\title{
Optimum Voxel Volume for Maximizing Contrast-to-Noise Ratio in BOLD Functional MRI
}

\author{
Norihiko Fujita*, $^{*}$, Kouji Okada ${ }^{2}$, Hisashi Tanaka $^{1}$ and Kenya Murase ${ }^{2}$ \\ ${ }^{I}$ Department of Radiology, Osaka University Graduate School of Medicine, Japan \\ ${ }^{2}$ Department of Medical Engineering, Osaka University Graduate School of Medicine, Japan
}

\begin{abstract}
Recent studies suggest that physiological noise, which determines the SNR of the fMRI time course at commonly used spatial resolutions, is largely spatially coherent. It is theoretically shown that the ratio of such a spatially coherent physiological to thermal noise determines the optimum voxel volume for maximizing BOLD CNR, relatively independent of the size of activation.
\end{abstract}

Keywords: MRI, BOLD, functional MRI, contrast-to-noise ratio, partial volume effects, physiological noise, optimization.

\section{INTRODUCTION}

In both anatomical [1] and functional MRI (fMRI) [2-4], voxel volume is one of the major imaging parameters for determining contrast-to-noise ratio (CNR). In general, use of a small voxel is desirable to minimize partial voluming effects (PVE), because it yields improved parcellation of anatomic and functional information. Improving spatial resolution may reduce PVE and therefore lead to an increase in the signal difference among tissues in anatomical MRI or between active and resting states in fMRI, but it inevitably results in a decrease in the signal-to-noise ratio (SNR). The optimum voxel volume for maximizing CNR is therefore generally determined as a compromise between the two opposing effects of PVE and SNR.

In the context of blood oxygenation level-dependent (BOLD) [5] contrast fMRI, a previous study by Yoo et al. [3] applied this basic principle. They theoretically showed that on the assumption that thermal noise is the only noise source, BOLD CNR is maximized when the size of the smoothing kernel or the voxel volume matches the volume of activation. The result obtained by taking into account only thermal noise is compatible with a general rule according to image processing theory. The maximum signal detection occurs when the size of the smoothing kernel approaches the spatial extent of the target signal [6]. On the other hand, a number of studies have shown that with the use of higher field strengths [7,8] and/or highly sensitive multichannel coils $[9,10]$, physiological noise dominates the SNR of fMRI time-course data at commonly used spatial resolutions.

The physiological noise mainly results from physiological fluctuations in the basal cerebral metabolism and hemodynamic state, and its major component with low frequencies of less than $0.1 \mathrm{~Hz}$ has spatial coherence over the range of voxels typically used for fMRI $[11,12]$. This implies that

*Address correspondence to this author at the Department of Radiology, Osaka University Graduate School of Medicine, 2-2 Yamadaoka, Suita, Osaka 565-0871, Japan; Tel: 81-6-6879-3434; Fax: 81-6-6879-3439;

E-mail: nofujita@radiol.med.osaka-u.ac.jp the magnitude of physiological noise relative to the baseline signal does not depend on voxel size so much because of its spatially coherent characteristics, which was shown in a recent experimental study by Triantafyllou et al. [13].

It appears obvious that the optimum voxel volume for maximizing BOLD CNR depends on the volume of activation, which is generally not known a priori. In this study, we show theoretically that when the effect of spatially coherent physiological noise in BOLD fMRI is taken into account, the voxel volume for maximizing BOLD CNR is relatively independent of the volume of activation, but is primarily determined by the ratio of physiological to thermal noise.

\section{BOLD CNR MODEL}

BOLD CNR is defined as the signal difference $(\Delta S)$ between active and resting states in units of the standard deviation $(\sigma)$ of the time-course fMRI signal of each voxel. When these quantities, $\Delta S$ and $\sigma$, are normalized to the baseline signal $(S)$, BOLD CNR is expressed by:

$\Delta S / \sigma=$ BOLD signal change $/{ }^{(\mathrm{n})} \sigma$,

where 'BOLD signal change' represents $\Delta S / S$ and ${ }^{(\mathrm{n})} \sigma$ is the normalized signal variation, $\sigma / S$, which corresponds to the reciprocal of the temporal SNR.

Noise in BOLD fMRI can be classified as thermal noise $\left(\sigma_{\mathrm{T}}\right)$, noise related to scanner instabilities $\left(\sigma_{\mathrm{I}}\right)$, and physiological noise $\left(\sigma_{\mathrm{P}}\right)$ [7]. Previous studies $[7,8]$ have demonstrated that $\sigma_{\mathrm{I}}$ is usually small compared with the other noise contributions on a well-adjusted MR scanner, although scanner stability may be somewhat variable over time and between scanners [14]. In this case, the time-course signal variation, ${ }^{(\mathrm{n})} \sigma$, is related to its thermal $\left({ }^{(\mathrm{n})} \sigma_{\mathrm{T}}: \sigma_{\mathrm{T}} / S\right)$ and physiological $\left({ }^{(\mathrm{n})} \sigma_{\mathrm{P}}: \sigma_{\mathrm{P}} / S\right)$ components. Taking into account the differences in voxel volume $(V)$ dependence between ${ }^{(\mathrm{n})} \sigma_{\mathrm{T}}$ and ${ }^{(\mathrm{n})} \sigma_{\mathrm{P}}$, the time-course signal variation, ${ }^{(\mathrm{n})} \sigma(V)$, is given by: 
${ }^{(\mathrm{n})} \sigma(V)=\left({ }^{(\mathrm{n})} \sigma_{\mathrm{T}}(V)^{2}+{ }^{(\mathrm{n})} \sigma_{\mathrm{P}}{ }^{2}\right)^{1 / 2}$.

The thermal noise contribution, $\sigma_{\mathrm{T}}$ is usually estimated by the SD of background noise or the signal statistics of a difference image [15], and then the physiological noise, $\sigma_{P}$ is assessed as the residual noise contribution to the total noise, $\sigma$, as shown in eq. (2).

In eq. (2), the physiological noise contribution, ${ }^{(n)} \sigma_{\mathrm{P}}$, was assumed to be independent of voxel volume [13]. Recent studies also support this assumption in the case of gradientecho fMRI data at commonly used spatial resolutions, although it may not be applicable to spin-echo fMRI data [16, 17]. It is also noted that for gradient-echo fMRI data at high resolutions of less than $8 \mathrm{~mm}^{3}$, the spatially correlated characteristics of the physiological noise are incomplete and partly affected by voxel volume [18]. The thermal noise contribution, ${ }^{(\mathrm{n})} \sigma_{\mathrm{T}}(V)$, is inversely proportional to voxel volume, $V$, for initial settings of MR imaging parameters, while ${ }^{(n)} \sigma$ ${ }_{\mathrm{T}}(V)$ is inversely proportional to the square root of voxel volume, $V^{1 / 2}$, for postprocessing acquired MR images [1].

In previous studies [19, 20], researchers measured the changes in BOLD signal at different spatial resolutions and found that the use of a higher spatial resolution could increase the magnitude of BOLD signal changes in activated areas. Such dependence of BOLD contrast on voxel volume is generally ascribed to PVE. The voxel volume generally determines the PVE, but the PVE in BOLD fMRI are also affected by the spatially smearing effect of the hemodynamic response and the specifics of fMRI experiment such as the field strength and gradient-echo or spin-echo signal acquisition [21]. In this study, we did not consider such detailed issues of BOLD PVE and examined the effect of varying voxel volume on BOLD contrast using a simple numerical model and $k$-space analysis method as described by Yoo et al. [3] (see Appendix). Assuming a three-dimensional Gaussian distribution of BOLD signal (signal at the origin, $B_{0}$; and full width at half maximum in each dimension, $L_{B}$ ), the modulation of BOLD signal $(B)$ due to the variation in voxel volume is described by:

$B=B_{0} \cdot F\left(\frac{V_{B}}{V}\right)$,

where

$$
\begin{aligned}
& F\left(\frac{V_{B}}{V}\right) \\
& =\alpha^{3} \mathrm{~V}_{\mathrm{B}} / \mathrm{V}\left(\int_{-1}^{1} \exp \left(-\pi \alpha^{2}\left(\frac{V_{B}}{V}\right)^{2 / 3} k^{2}\right) d k\right)^{3} .
\end{aligned}
$$

In eq. (4), $\alpha$ is a constant and $V_{B}$ is $L_{B}{ }^{3}$, which corresponds to a characteristic volume for the hypothetical BOLD activation, and $F\left(V_{B} / V\right)$ represents the relative BOLD contrast modulation due to PVE (Fig. (1); See Appendix).

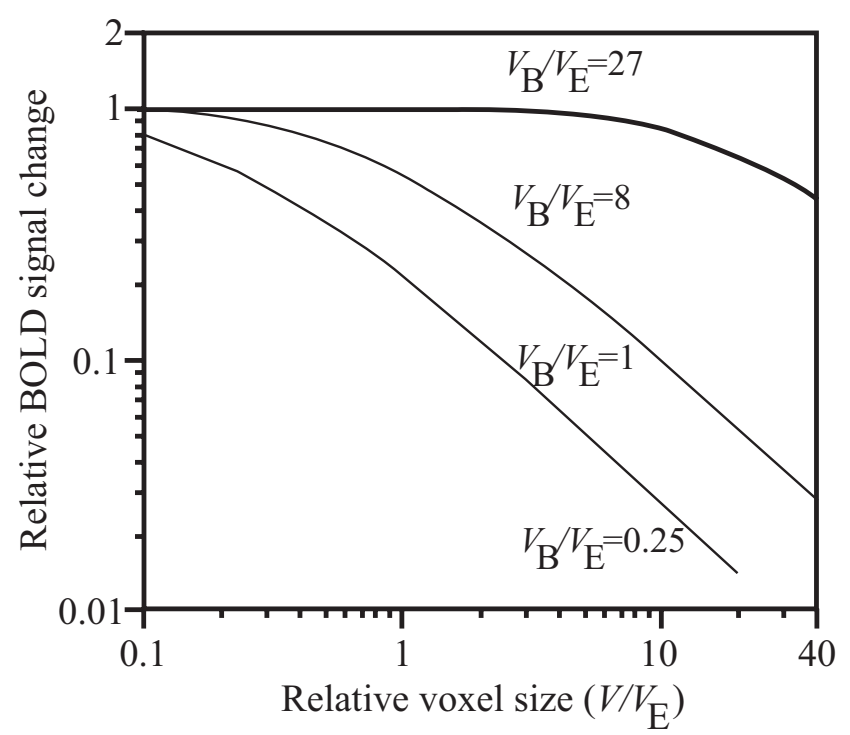

Fig. (1). BOLD contrast modulation due to PVE. A threedimensional Gaussian distribution of BOLD signal was assumed. $V_{\mathrm{B}}$ represents a characteristic volume for the hypothetical activation, and $V_{\mathrm{E}}$ a reference voxel volume. The abscissa represents a voxel volume relative to $V_{\mathrm{E}}, V / V_{\mathrm{E}}$, and each value of $V_{\mathrm{B}} / V_{\mathrm{E}}$ represents the activation volume relative to $V_{\mathrm{E}}$. The function representing PVE of BOLD contrast takes a constant value of 0.54 when the voxel volume matches the activation volume, i.e., $V=V_{\mathrm{B}}$.

To evaluate BOLD CNR as a function of voxel volume, we introduced a reference voxel volume, $V_{\mathrm{E}}$. We defined the magnitude of thermal noise at $V=V_{\mathrm{E}}$ as equivalent to that of physiological noise, i.e., ${ }^{(\mathrm{n})} \sigma_{\mathrm{T}}\left(V_{\mathrm{E}}\right)={ }^{(\mathrm{n})} \sigma_{\mathrm{P}}$. Then, eq. (2) is represented by a function of the relative voxel volume, $V / V_{E}$, as follows:

${ }^{(\mathrm{n})} \sigma={ }^{(\mathrm{n})} \sigma_{\mathrm{P}} \cdot G\left(\frac{V}{V_{E}}\right)$,

where

$$
\begin{aligned}
& G\left(\frac{V}{V_{E}}\right)=\left(\frac{V_{E}}{V}+1\right)^{1 / 2} \text { for }^{(\mathrm{n})} \sigma_{\mathrm{T}}(V) \propto 1 / V^{1 / 2} \\
& =\left(\left(\frac{V_{E}}{V}\right)^{2}+1\right)^{1 / 2} \text { for }^{(\mathrm{n})} \sigma_{\mathrm{T}}(V) \propto 1 / V .
\end{aligned}
$$

The BOLD CNR, which is defined by the ratio of the BOLD signal, $B$ in eq. (3), to the total noise, ${ }^{(n)} \sigma$ in eq. (5), is given by:

$\operatorname{BOLD~CNR}=B_{0} /{ }^{(\mathrm{n})} \sigma_{\mathrm{P}} \cdot F\left(\frac{V_{B}}{V_{E}} \quad \frac{V_{E}}{V}\right) / G\left(\frac{V}{V_{E}}\right)$.

In eq. (8), the independent variable of the function that represents PVE of BOLD contrast, $F$, was expressed by $V_{\mathrm{B}} / V_{\mathrm{E}} \cdot V_{\mathrm{E}} / V$ rather than $V_{\mathrm{B}} / V$ in eq. (3). The parameter $V_{\mathrm{B}} / V_{\mathrm{E}}$ represents the activation volume relative to the reference voxel volume. As evident from eq. (8), the BOLD CNR is 
determined by $F\left(V_{\mathrm{B}} / V_{\mathrm{E}} \cdot V_{\mathrm{E}} / V\right) / G\left(V / V_{\mathrm{E}}\right)$ for fixed values of BOLD signal change $\left(B_{0}\right)$ and physiological noise $\left({ }^{(\mathrm{n})} \sigma_{\mathrm{P}}\right)$. We therefore examined the voxel volume dependence of BOLD CNR by numerically evaluating this function that provides the relative magnitude of BOLD CNR. The numerical results are shown as a function of the relative voxel volume, $V / V_{\mathrm{E}}$, for given values of the relative activation volume, $V_{\mathrm{B}} / V_{\mathrm{E}}$.

\section{RESULTS}

For comparison, we first describe the calculated results of BOLD CNR when only thermal noise was taken into account, i.e., ${ }^{(\mathrm{n})} \sigma_{\mathrm{P}}=0$. For this calculation, we set the reference voxel volume, $V_{\mathrm{E}}$, as ${ }^{(\mathrm{n})} \sigma_{\mathrm{T}}\left(V_{\mathrm{E}}\right)=1 \%$. Then, the noise function, $G\left(V / V_{\mathrm{E}}\right)$, is simply given by $\left(V_{\mathrm{E}} / V\right)^{1 / 2}$ and $V_{\mathrm{E}} / V$, corresponding to eqs. (6) and (7), respectively. For $\mathrm{G}\left(V / V_{\mathrm{E}}\right)$ $=\left(V_{\mathrm{E}} / V\right)^{1 / 2}$, BOLD CNR takes a peak value at $V / V_{\mathrm{E}}=V_{\mathrm{B}} / V_{\mathrm{E}}$, i.e., $V=V_{\mathrm{B}}$, which means that BOLD CNR is maximized when the voxel volume matches the volume of activation (Fig. 2a).
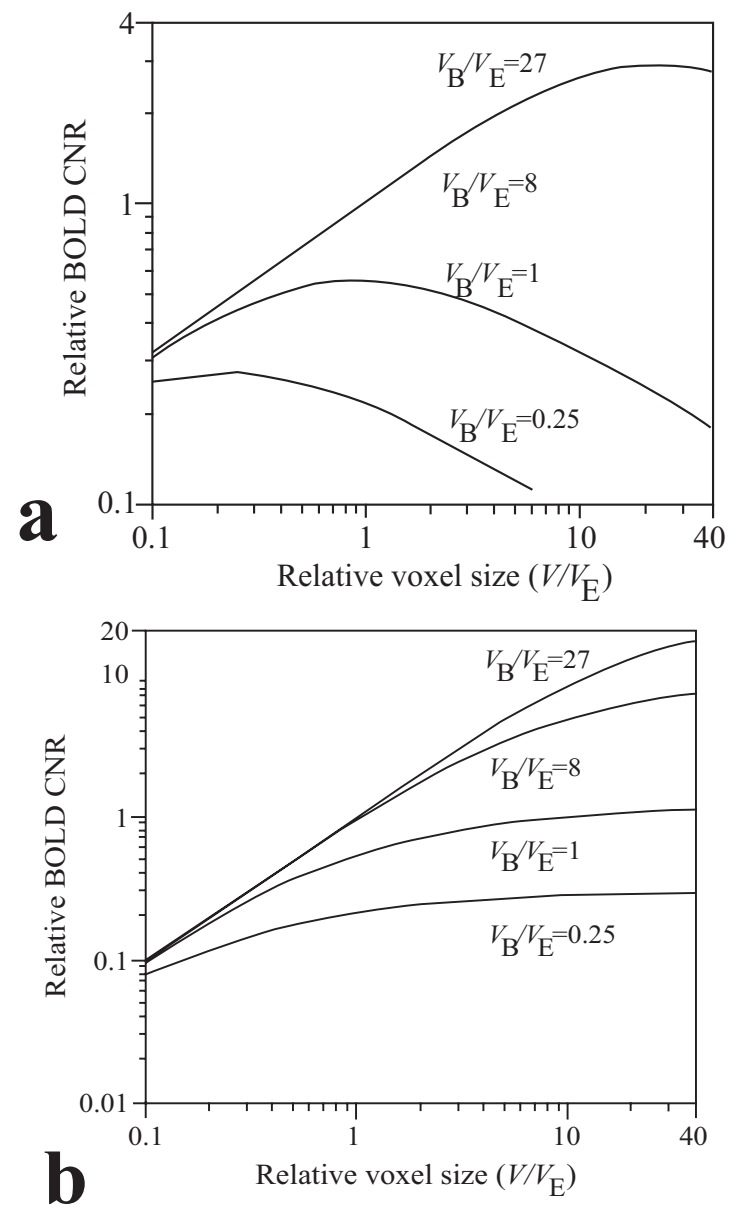

Fig. (2). BOLD CNR in the presence of only thermal noise. The abscissa represents a voxel volume relative to $V_{\mathrm{E}}, V / V_{\mathrm{E}}$. The relative BOLD CNR values were calculated as the ratio of the BOLD contrast modulation shown in Fig. (1) to the noise function, $G\left(V / V_{\mathrm{E}}\right)$, in eq. (8). When thermal noise alone is considered, $G\left(V / V_{\mathrm{E}}\right)$ is simply given by $\left(V_{\mathrm{E}} / V\right)^{1 / 2}$ for the case of spatial filtering of acquired MR images (Fig. 2a), and $V_{\mathrm{E}} / V$ for the case of initial imaging parameter settings for acquiring MR images (Fig. 2b).
This theoretical prediction, obtained using the threedimensional BOLD activation model, is conceptually the same as that obtained using a one-dimensional model [3]. For $\mathrm{G}\left(V / V_{\mathrm{E}}\right)=V_{\mathrm{E}} / V$, BOLD CNR increases almost linearly with voxel volume until $V=V_{B}$, after which it asymptotically approaches a constant value, depending on the activation volume, $V_{B} / V_{E}$ (Fig. 2b). This result also indicates that the optimal voxel volume matches the volume of activation, because it represents the minimum voxel volume with nearly the maximum of the available BOLD CNR.

Fig. (3) shows the voxel volume dependence of BOLD CNR in the presence of physiological noise. Figs. 3a and $\mathbf{3 b}$ correspond to the cases of ${ }^{(\mathrm{n})} \sigma{ }_{\mathrm{T}}(V) \propto 1 / V^{1 / 2}$ and ${ }^{(\mathrm{n})} \sigma$ $\mathrm{T}_{\mathrm{T}}(V) \propto 1 / V$, respectively. In the regime of $V / V_{\mathrm{E}}<1$, where the thermal noise contribution dominates the total noise, the numerical results of BOLD CNR in the presence of physiological noise are almost the same as those shown in Figs. 2a and $\mathbf{2 b}$, respectively. Distinct differences were noted when the relative voxel volume, $V / V_{\mathrm{E}}$, is comparable with or greater than 1 , where the physiological noise significantly contributes to or dominates the total noise.
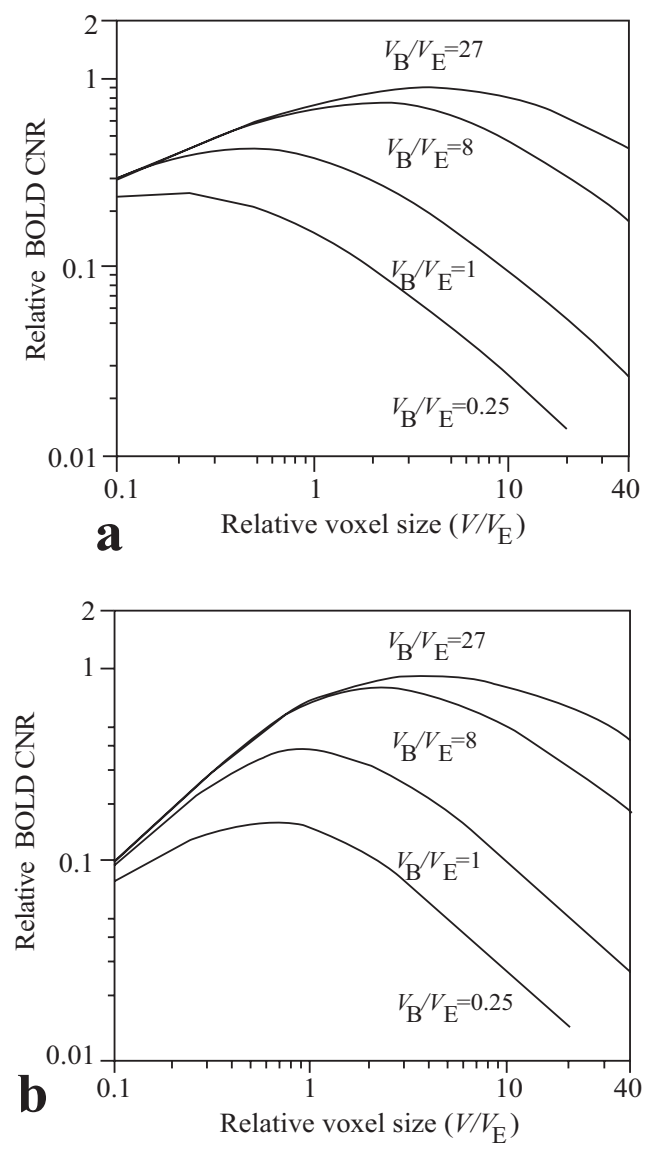

Fig. (3). BOLD CNR in the presence of physiological noise. The abscissa represents a voxel volume relative to $V_{\mathrm{E}}, V / V_{\mathrm{E}}$. Taking into account the presence of physiological noise, the noise function $G\left(V / V_{\mathrm{E}}\right)$ is given by $\left(V_{\mathrm{E}} / V+1\right)^{1 / 2}$ for the case of spatial filtering of acquired MR images (Fig. 3a) and $\left(\left(V_{\mathrm{E}} / V\right)^{2}+1\right)^{1 / 2}$ for the case of initial imaging parameter settings for acquiring MR images (Fig. 3b). Note that the relative BOLD CNR values do not exceed unity, and that the scale of the ordinate is therefore set differently from that in Fig. (2). 
Regarding the case of variation in the spatial resolution for acquired MR images, i.e., ${ }^{(\mathrm{n})} \sigma_{\mathrm{T}}(V) \propto 1 / V^{1 / 2}$, we compare the effects of the absence (Fig. 2a) and presence (Fig. 3a) of physiological noise on BOLD CNR. In contrast to Fig. 2a, the relative BOLD CNR does not exceed unity, irrespective of the activation volume (Fig. 3a). For relative activation volumes of $V_{\mathrm{B}} / V_{\mathrm{E}}=1,8$, and 27, maximal BOLD CNR values were obtained at $V / V_{\mathrm{E}}=0.7,2$, and 5, respectively, rather than at $V / V_{\mathrm{E}}=1,8$, and 27, as shown in Fig.2a. For the smallest activation volume of $V_{\mathrm{B}} / V_{\mathrm{E}}=0.25$, the peak BOLD CNR occurred commonly at $V / V_{\mathrm{E}}=0.25$.

Regarding the case of the initial settings of spatial resolution for acquiring MR images, i.e., ${ }^{(\mathrm{n})} \sigma \mathrm{T}_{\mathrm{T}}(V) \propto 1 / V$, the behavior of BOLD CNR in the presence of physiological noise (Fig. 3b) is distinctively different from that in the absence of physiological noise (Fig. 2b), while it is similar to that shown in Fig. 3a. The relative BOLD CNR calculated does not exceed unity, irrespective of the activation volume. For relative activation volumes of $V_{\mathrm{B}} / V_{\mathrm{E}}=0.25,1,8$, and 27, maximal BOLD CNR values were obtained at $V / V_{\mathrm{E}}$ of approximately $0.7,1,2$, and 3 , respectively.

The asymptotic behaviors of BOLD CNR when $V / V_{\mathrm{E}}$ was much greater than 1 (Figs. 3a and $\mathbf{3 b}$ ) are similar to those of $F\left(V_{\mathrm{B}} / V_{\mathrm{E}} \cdot V_{\mathrm{E}} / V\right)$ (Fig. 1) that was assumed to represent PVE of BOLD contrast. This is because the noise function, $G\left(V / V_{\mathrm{E}}\right)$, takes an almost constant value of unity in this regime. The asymptotic behaviors of BOLD CNR would be somewhat different, depending on the specifics assumed in the evaluation of the effect of BOLD PVE, including the form of spatial distribution and the definition of a characteristic volume for the hypothetical activation. However, the BOLD CNR behaviors described above for the other regimes, where $V / V_{\mathrm{E}}$ is smaller than or comparable with 1 , would not be significantly affected by such minor differences in the evaluation of BOLD PVE.

\section{DISCUSSION}

As evident from eqs. (6) and (7), the gain in temporal SNR is only marginal when the voxel volume is increased beyond the reference voxel volume, $V_{\mathrm{E}}$, at which the physiological and thermal noise contributions are equal. In the context of the applications of parallel imaging to fMRI, a similar SNR consideration based on the ratio of physiological to thermal noise has been shown to be effective for the optimum choice of acceleration factor [10]. Such an SNR consideration can also provide $V_{\mathrm{E}}$ as an upper limit to voxel volume in fMRI $[13,22]$. However, it is obvious that an optimum voxel volume in fMRI should be determined by taking into account the two opposing effects as a function of voxel size, BOLD PVE and temporal SNR.

The BOLD CNR simulation results in the absence of physiological noise (Figs. 2a and 2b) significantly varied, depending on the volume of activation. Thus, the volume of activation, which is generally not known a priori, determines the optimum voxel volume. The presence of physiological noise significantly reduced the activation volume dependency, the relative BOLD CNR was maximized at a limited range of voxel volumes between the reference volume, $V_{\mathrm{E}}$, and each volume of activation, being either small or large compared to $V_{\mathrm{E}}$ (Figs. 3a and 3b). This suggests that $V_{\mathrm{E}}$ corresponds to the optimum voxel volume at which a sufficiently high BOLD CNR is warranted for a wide range of BOLD activation volumes. The tendency was more prominent when setting the spatial resolution to be used for acquiring MR images, as evident from the comparison of Figs. 3a and $\mathbf{3 b}$. This indicates that the initial choice of a voxel volume in fMRI significantly affects the detectability of functional activation, which has been shown in a recent experimental fMRI study [4].

From experimental data using a conventional volume head coil [13], $V_{\mathrm{E}}$ for gray matter was estimated to be about $50(3.7 \times 3.7 \times 3.7) \mathrm{mm}^{3}$ at $1.5 \mathrm{~T}$ and $20(2.7 \times 2.7 \times 2.7)$ $\mathrm{mm}^{3}$ at $3 \mathrm{~T}$. We performed a similar measurement at $1.5 \mathrm{~T}$ and found that $V_{\mathrm{E}}$ was 45 to $50 \mathrm{~mm}^{3}$ for gray matter regions (data not shown). On the assumption that the optimum voxel volume is given by $V_{\mathrm{E}}$, this volume of $V_{\mathrm{E}}$ at $1.5 \mathrm{~T}$ agrees well with the optimum voxel volume of $80 \mathrm{~mm}^{3}$ experimentally determined for the detection of motor cortex activation under similar experimental conditions [23], considering that the optimum voxel volumes are situated on relatively broad curves (Figs. 3a and 3b).

With the use of higher field strengths and/or highly sensitive multichannel coils, $V_{\mathrm{E}}$ can be significantly decreased $[13,22]$. In particular, a recent study at $3 \mathrm{~T}$ with a highly sensitive 16-channel array coil [22] showed that $V_{\mathrm{E}}$ for gray matter was estimated to be $(1.8 \mathrm{~mm})^{3}$, which approximately matches the cortical thickness and thus may optimize BOLD contrast by minimizing PVE [24]. On the other hand, cognitive neuroscience applications of fMRI in the context of multiple-subject analysis tend to apply a Gaussian filter of 5-10 $\mathrm{mm}$ in width during postprocessing to project the data to a scale, where homologies in functional anatomy are expressed across subjects, and hence the effective voxel volume usually exceeds $100 \mathrm{~mm}^{3}$. The optimum voxel volume as determined by $V_{\mathrm{E}}$ may therefore be small enough for such cognitive neuroscience applications, even at $1.5 \mathrm{~T}$.

In this study, we did not consider the effects of susceptibility artifacts, which may lead to a considerable signal loss at large voxel dimensions. In certain regions such as the temporal lobe, use of a voxel size smaller than $V_{\mathrm{E}}$ may therefore be preferable, depending on the severity of susceptibility artifacts $[25,26]$. Although use of a shorter $T E$ as well as smaller voxels generally mitigates the deteriorative effect of large susceptibility variation on gradient-echo signal, the optimum $T E$ for maximizing BOLD fractional signal change is equal to the $\mathrm{T} 2 *$ of gray matter [27] and hence is usually set very long. This may indicate that BOLD CNR is maximized when $T E=T 2 *$; however, this holds when only thermal noise is considered. In contrast, Hyde et al. [24] demonstrated that BOLD CNR is independent of $T E$ when physiological noise of BOLD origin dominates other noise. Therefore, the contribution of physiological noise relative to thermal noise significantly affects the choice of $T E$ as shown in a recent study by $\mathrm{Wu}$ and $\mathrm{Li}$ [28], as well as the choice of voxel volume as shown in this study. Taking into account 
both effects, it is suggested that for a voxel volume smaller than $V_{\mathrm{E}}$, a rela-tively long $T E$ comparable with tissue $T 2 *$ is optimal for maximizing BOLD CNR, while for a bigger voxel, a shorter TE would be better for mitigating susceptibility artifacts.

In this study, the physiological noise contribution was assumed to be spatially correlated and hence voxel volume independent (Fig. 3), instead of spatially uncorrelated and hence independent. The results in the presence of thermal noise alone (Fig. 2) may be applicable if the physiological noise can be described by a Gaussian distribution and is spatially correlated in the same way as thermal noise is. Several studies $[13,16,17]$ also support this assumption in the case of gradient-echo fMRI data at commonly used spatial resolutions. However, a recent study at high spatial resolutions [18] showed that the extent to which physiological noise is spatially correlated lay between the two extreme cases, corresponding to Figs. $\mathbf{2}$ and $\mathbf{3}$, respectively. In this sense, the optimum voxel volume as defined by $V_{\mathrm{E}}$ may be more relevant to cognitive neuroscience applications at commonly used low spatial resolutions, as opposed to a noticeable trend towards high-resolution fMRI, in which the focus of brain mapping may shift from a simple detection of activity patterns as described in this study, to an analysis of the amount of information they convey [29].

\section{CONCLUSION}

If physiological noise predominates over thermal noise at a certain volume of voxel, use of a larger voxel leads to only a marginal decrease in total noise and yet may decrease the detectability of activation because of an increased PVE of BOLD signal. This phenomenon was examined theoretically and the results show that the highest BOLD CNR was observed at an intermediate voxel volume between the reference volume, $V_{\mathrm{E}}$, and each volume of activation, being either small or large compared to $V_{\mathrm{E}}$. Optimum voxel size in fMRI may therefore be selected in such a way that the magnitudes of physiological and thermal noise are comparable, relatively independent of the size of activation, which is generally not known a priori.

\section{APPENDIX}

MRI raw image data are generally acquired as $k$-space data, and hence the signal modulation that accompanies the change in spatial resolution is estimated as the corresponding change in $k$-space size [3]. We consider a one-dimensional Gaussian distribution of the BOLD signal, $\mathrm{f}(x)=B_{0} \exp (-$ $\left.4(\log 2) x^{2} / L_{\mathrm{B}}{ }^{2}\right)$, where $B_{0}$ is the BOLD signal at the origin and $L_{\mathrm{B}}$ is the full width at half maximum. Fourier transform of this function is also expressed in the form of a Gaussian function, $F(k)=2 \alpha B_{0} L_{\mathrm{B}} \exp \left(-\pi\left(2 \alpha L_{\mathrm{B}} k\right)^{2}\right)$, where $\alpha$ is $\sqrt{\pi / \log 2} / 4$. For a given voxel size of $L_{\mathrm{x}}$ in the $x$ dimension, the frequency bandwidth in $k$-space is limited within the range of $\left[1 / 2 L_{\mathrm{x}},-1 / 2 L_{\mathrm{x}}\right]$. Due to PVE, BOLD signal at the origin $(B)$ decreases with the increase of $L_{\mathrm{x}}$ and is represented as the integral of $F(k)$ in the corresponding $k$ space range of $\left[1 / 2 L_{\mathrm{x}},-1 / 2 L_{\mathrm{x}}\right]$ :
$B=B_{0} \cdot F\left(L_{\mathrm{B}} / L_{\mathrm{x}}\right)$

where

$F\left(L_{\mathrm{B}} / L_{\mathrm{x}}\right)=\alpha L_{\mathrm{B}} / L_{\mathrm{x}} \int_{-1}^{1} \exp \left(-\pi \alpha^{2}\left(\frac{L_{B}}{L_{x}}\right)^{2} k^{2}\right) d k$.

The function, $F\left(L_{\mathrm{B}} / L_{\mathrm{x}}\right)$, represents BOLD PVE in one dimension, while eq. (4) in the text corresponds to its threedimensional version.

\section{REFERENCES}

[1] Edelstein WA, Glover GH, Hardy CJ, Redington RW. The intrinsic signal-to-noise ratio in NMR imaging. Magn Reson Med 1986; 3 : 604-18.

[2] Lowe MJ, Sorenson JA. Spatially filtering functional magnetic resonance imaging data. Magn Reson Med 1997; 37: 723-9.

[3] Yoo SS, Guttmann CRG, Panych LP. Multiresolution data acquisition and detection in functional MRI. Neuroimage 2001; 14: 147685 .

[4] Scouten A, Papademetris X, Constable RT. Spatial resolution, signal-to-noise ratio, and smoothing in multi-subject functional MRI studies. Neuroimage 2006; 30: 787-93.

[5] Ogawa S, Lee TM, Kay AR, Tank DW. Brain magnetic resonance imaging with contrast dependent on blood oxygenation. Proc Natl Acad Sci USA 1990; 87: 9868-72.

[6] Ludeman LC. Random processes: filtering, estimation, and detection. John Wiley \& Sons: Hoboken (NJ) 2002.

[7] Krueger G, Glover GH. Physiological noise in oxygenation-sensitive magnetic resonance imaging. Magn Reson Med 2001; 46: 6317.

[8] Krueger G, Kastrup A, Glover GH. Neuroimaging at 1.5T and 3.0T: comparison of oxygenation-sensitive magnetic resonance imaging. Magn Reson Med 2001; 45: 595-604.

[9] de Zwart JA, van Gelderen P, Kellman P, Duyn JH. Application of sensitivity encoded echo planar imaging for blood oxygen leveldependent functional brain imaging. Magn Reson Med 2002; 48 : 1011-20.

[10] Pinkerton RG, McKinnon GC, Menon RS. SENSE optimization of a transceive surface coil array for MRI at $4 \mathrm{~T}$. Magn Reson Med 2006; 56: 630-6.

[11] Zarahn E, Aguirre GK, D'Esposito M. Empirical analyses of BOLD fMRI statistics I. Spatially unsmoothed data collected under null-hypothesis conditions. Neuroimage 1997; 5: 179-97.

[12] Kamba M, Sung AW, Ogawa S. A dynamic system model-based technique for functional MRI data analysis. Neuroimage 2004; 22 : 179-87.

[13] Triantafyllou C, Hoge RD, Krueger G, Wiggins CJ, Potthast A, Wiggins GC, Wald LL. Comparison of physiological noise at 1.5 $\mathrm{T}, 3 \mathrm{~T}$, and $7 \mathrm{~T}$ and optimization of fMRI acquisition parameters. Neuroimage 2005; 26: 243-50.

[14] Friedman L, Glover GH. Report on a multicenter fMRI quality assurance protocol. J Magn Reson Imaging 2006; 23: 827-39.

[15] Dietrich O, Raya JG, Reeder SB, Reiser MF, Schoenberg SO. Measurement of signal-to-noise ratios in MR images: influence of multichannel coils, parallel imaging, and reconstruction filters. J Magn Reson Imaging 2007; 26: 376-85.

[16] Yacoub E, Van der Moortele PF, Shmuel A, Ugurbil K. Signal and noise characteristics of Hahn SE and GE BOLD fMRI at 7T in humans. Neuroimage 2005; 24: 738-50.

[17] Liu CSJ, Miki A, Hulvershorn J, Bloy L, Gualtieri EE, Liu GT, Leigh JS, Haselgrove JC, Elliott MA. Spatial and temporal characteristics of physiological noise in fMRI at 3T. Acad Radiol 2006; 13: 313-23.

[18] Triantafyllou C, Hoge RD, Wald LL. Effect of spatial smoothing on physiological noise in high-resolution fMRI. Neuroimage 2006; 32: 551-7.

[19] Frahm J, Merboldt KD, Haenicke W. Functional MRI of human brain activation at high spatial resolution. Magn Reson Med 1993; 42: 1014-8.

[20] Hoogenraad FGC, Hofman MBM, Pouwels PJW, Reichenbach JR, Rombouts SARB, Haacke EM. Sub-millimeter fMRI at 1.5 Tesla: 
correlation of high-resolution with low-resolution measurement. J Magn Reson Imaging 1999; 9: 475-82.

[21] Norris DG. Principles of magnetic resonance assessment of brain function. J Magn Reson Imaging 2006; 23: 794-807.

[22] Bodurka J, Ye F, Petridou N, Murphy K, Bandettini PA. Mapping the MRI voxel volume in which thermal noise matches physiological noise - Implications for fMRI. Neuroimage 2007; 34; 542-49.

[23] Glover GH, Krueger G. Optimum voxel size in BOLD fMRI: Proceedings of the 10th Annual Meeting of International Society for Magnetic Resonance in Medicine; Aug 2002: p. 1395. Available form: http://www.ismrm.org

[24] Hyde JS, Biswal BB, Jesmanowicz A. High-resolution fMRI using multislice partial k-space GR-EPI. Magn Reson Med 2001; 46: 114-25.

[25] Chen NK, Dickey CC, Yoo SS, Guttmann DRG, Panych LP. Selection of voxel size and slice orientation for fMRI in the presence of susceptibility field gradients: application to imaging of the amygdala. Neuroimage 2003; 19: 817-25

[26] Bellgowan PSF, Bandettini PA, van Gelderen P, Martin A, Bodurka J. Improved BOLD detection in the medial temporal region using parallel imaging and voxel volume reduction. Neuroimage 2006; 29: 1244-51.

[27] Ogawa S, Lee TM, Barrere B. The sensitivity of magnetic resonance image signals of a rat brain to changes in the cerebral venous blood oxygenation. Magn Reson Med 1993; 29: 205-10.

[28] Wu G and Li S-J. Theoretical noise model for oxygenationsensitive magnetic resonance imaging. Magn Reson Med 2005; 53 . 1046-54.

[29] Kriegeskorte N, Bandettini P. Analyzing for information, not activation, to exploit high-resolution fMRI. Neuroimage 2007; 38 : 649-62.

Received: March 12, 2008

Revised: April 30, 2008

Accepted: May 02, 2008

(C) Fujita et al.; Licensee Bentham Open.

This is an open access article distributed under the terms of the Creative Commons Attribution License (http://creativecommons.org/licenses/by/2.5/), which permits unrestrictive use, distribution, and reproduction in any medium, provided the original work is properly cited. 